\title{
Proper-Time Relativistic Dynamics and the Fushchych-Shtelen Transformation
}

\author{
Tepper L. GILL, James LINDESAY, M.F. MAHMOOD and W.W. ZACHARY \\ Computational Science and Engineering Research Center \\ and Department of Physics, Howard University \\ Washington, D.C. 20059
}

\begin{abstract}
We report on a new formulation of classical relativistic Hamiltonian mechanics which is based on a proper-time implementation of special relativity using a transformation from observer proper-time, which is not invariant, to system proper-time which is a canonical contact transformation on extended phase-space. This approach does not require the use of time as a fourth coordinate and so we prove that it satisfies the two postulates of special relativity. In the free particle case, our transformation theory generates a Poincaré group which fixes time (system proper-time). We prove that the Fushchych-Shtelen transformation is an element of our group, which fixes time for Maxwell's equations. In the interaction case, our transformation theory allows us to avoid the no-interaction theorem. We show that the Santilli Isotopes appear naturally when interaction is turned on.
\end{abstract}

\section{Background}

In his introductory paragraphs to the famous 1905 paper, Einstein made it clear that he was very concerned with the physical problem (inconsistency) of the asymmetry arising from Maxwell's electrodynamics when applied to bodies in relative motion. Einstein showed that with only two postulates, both very reasonable, he could remove the asymmetry and maintain the invariance of the electromagnetic field equations.

In his struggle with the nature of space and time, Einstein had gone far beyond what was normally considered theoretical physics. This caused a certain amount of confusion for many physicists, but the initial results were without question.

Beginning with Minkowski, Einstein's approach began to attract increased (positive) visibility among the general public and mathematicians in particular. The mathematics community, reeling from a shock wave of far reaching discoveries in algebra, analysis and geometry, was ripe for this new bundle of intellectual elegance coming from the very foundations of physical reality. Both the interest, help and support of the mathematics community played a major role in the time compression between the introduction of the special and general theories. By 1907, Einstein was concerned with a deeper and more difficult question, namely the possibility that the laws of nature could and should be independent of accelerating observers.

Copyright (c) 1997 by Mathematical Ukraina Publisher. All rights of reproduction in any form reserved. 


\section{Perspective}

We now recognize that scientific investigation is itself a social enterprise and as such, is not immune from the overall mood and attitude of the times. The turn of the century was a time of great excitement and expectation. Great advances were being made in many areas of mathematics, physics, philosophy and engineering. The corresponding impact on social and political expectations helped to create a general feeling that all problems could be solved in a fairly short time.

By 1905, Electrodynamics was the prima donna of theoretical physics. Newtonian mechanics was being rapidly reduced to practical applications by engineers and was losing its status in physics. In this atmosphere, it is obvious that any problems at the interface between the Newtonian and Maxwell theories would be resolved in favor of electrodynamics.

It is interesting to note that virtually no one in the physics community had serious problems with the postulates of special relativity. Initially, Lorentz $[1,23]$ was concerned with the lack of an "absolute" time variable. This attitude was the motivation for the works of Ritz and Tolman [1, 8, 24, 25]. Poincaré noted [26, 27] that one approach to the implementation of the Lorentz-invariant requirement was to make time a fourth imaginary coordinate so that Lorentz transformations became rotations in this system. Poincaré knew that geometry was derived from an abstraction of the general representation of the relationships between physical bodies and as such, did not consider this implementation fundamental or profound. Minkowski's pronouncement that we should indeed take the idea of time as a fourth coordinate with real physical and philosophical implications seemed to give this approach the impetus needed to acquire widespread acceptance.

\section{Purpose}

The purpose of this paper is to report on recent progress on a new implementation of the first two explicit postulates of the special theory of relativity. This implementation is based on the realization that all observers identify a unique, invariant "proper" time variable associated with the observed system. Hence they need not use their own "propertime clock" to describe the system dynamics which is not invariant for all observers. Our implementation recognizes that the canonical Hamiltonian is the generator of time translations and hence, any change in the time variable should lead to its translation generator. We are thus lead to treat the transformation from observer (proper) time to system (proper) time as a canonical contact transformation on extended phase space. This approach automatically provides the canonical Hamiltonian and it is easy to prove that the phase flow (Poincaré-Cartan invariant) is preserved.

The subject of this paper is motivated by discussions of one of us (T.L.G) with Professor Fuchchych concerning his discovery of a Galilean transformation group for Maxwell's equations which leaves the time variable invariant $[12,22]$. We both agreed that there must be some relationship between his transformation group and the invariance group for our theory.

In section 1, we begin with a brief introduction using an intuitive and mathematically simple approach. We prove that our approach preserves the Poincaré-Cartan invariant on extended phase space, identify the generator and prove that our theory satisfies the 
first two postulates. We then show that the Fushchych-Shtelen theorem is an (important) special case. We finally prove that our theory avoids the famous no-interaction theorem. In section 2, we pause to study the issue of interaction more carefully and show how Santilli's Lie-isotopic methods become natural in this case. We provide a few standard examples so that we can study the interaction many-particle case in more detail in section 3. In section 3 , we prove that our theory has the cluster decomposition property. We use this result to prove that the universe has a unique (observable) proper-clock and canonical Hamiltonian (of course no preference is implied). In conclusion we identify some physical and mathematical problems which need to be addressed.

\section{Problems}

It is unfortunate that the founding fathers, Einstein, Lorentz, Poincaré and others, did not have the intellectual leisure to study and contemplate the implications associated with Minkowski's re-interpretation of Poincare's mathematical device as a real physical necessity; namely, that time be treated as a fourth coordinate and the mathematical representation be constrained to satisfy the geometric implementation of special relativity. Although not recognized at the time, it is now clear that this approach is equivalent to a third postulate (see [1]). This third postulate has not been nearly as successful as the general scientific community has been led to believe. There are problems, both physical and mathematical, associated with this implementation which, as shown in [1], have nothing to do with the two postulates of Einstein.

In this formalism, the one-particle theory is easy to implement and is essentially trivial (however, there is a problem). On the other hand, the many-particle theory has yet to be formulated in a manner that lives up to a minimal set of physically reasonable requirements. For example, any observer can write down a center of "mass" relationship for a system of non-interacting particles. However, it was shown by Pryce [2] that the most natural choices for the position are not the vector parts of a four-vector. Indeed, he has shown that there does not even exist a relativistic analog of the (Newtonian) reduction of the two- body problem (Rohrlich [3], Dirac [4], Rosen [5]). Goldstein noted [6] that there is not (and can never be) a relativistic rigid body theory. Things became critical after 1963 when Currie et al [7] showed that the minimal requirements for a relativistic quantum mechanics: Hamiltonian representation, canonical independent variables and invariance under the Lorentz group, was only compatible with non-interacting particles. Since then, this result has become known as the no-interaction theorem (see [8] for other attempts, problems, and references along this line).

At the very beginning, we are confronted by what can only be called physical nonsense. The four-velocity is defined as $u_{1}=\frac{c}{\sqrt{1-\beta^{2}}}, \quad u_{i}=\frac{v_{i}}{\sqrt{1-\beta^{2}}}$ so that $u_{\mu} u^{\mu}=-c^{2}$. Students are told (and others apparently believe) that this result insures that no object has a speed larger than that of light. This is clearly a mathematical four-vector on Minkowski space but it is also not physical. If we consider an electron moving at say .916c along the $\mathrm{y}$-axis. It is easy to see that $u_{1}=2.29 c, u_{y}=2.50 c$ so that our constraint $u_{\mu} u^{\mu}=-c^{2}$, is satisfied (within round off) but this result does not satisfy the original purpose and is unphysical. The problem is obvious and transparent, $u_{\mu}=\frac{d x_{\mu}}{d \tau}$ (with $\tau$ the proper time) can take on values greater than c. It is the constraint condition which is the problem 
(since $\frac{d x_{\mu}}{d t} \leq c$ ) and is only required because of the third postulate.

Using units in which $c=1$, the fourth component is $\frac{d t}{d \tau}$. Using $\sqrt{1-\beta^{2}}=\frac{m}{H}$ and $H=\sqrt{p^{2}+m^{2}}$, it is easy to see that: $\frac{d t}{d \tau}=\frac{\sqrt{p^{2}+m^{2}}}{m}$. It follows that on the natural transition to quantum mechanics, we get that:

$$
\frac{d t}{d \tau}=\frac{\sqrt{-\hbar^{2} \Delta+m^{2}}}{m} .
$$

This leads to at least two important observations:

1. We cannot treat proper time as a parameter (in quantum theory) without physical and mathematical justification.

2. We cannot treat proper-time as a 5 th variable independent of its direct relationship with $t$.

Even in the one-particle implementation, the use of proper time in the current implementation is problematic. This is because there is no specified or derived relationship between $\tau$ and $H$. After all, $t$ and $H$ are canonical variables so that on physical and methodological grounds, the canonical Hamiltonian associated with $\tau$ should be produced (and used). The fact that this is not done, makes the one-particle theory suspect.

It has recently been shown by Whitney [9-11] that the Lienard-Wiechert potentials are neither covariant nor manifestly covariant as they appear to be, due to the retardation in their construction. This means, among other things, that the use of these potentials in the interaction representation of classical and/or quantum theory does not make these formulations covariant as they appear. This problem shows that the use of formalism without detailed physical and methodological analysis can easily lead to erroneous "procedures" and/or conclusions. For example, the above result implies that quantum electrodynamics (despite its successes) requires a serious methodological review.

At a more fundamental level, Fushchych and Shtelen have shown that the free Maxwell's equations have an additional invariance group [12] which is "Galilean". This group leads to different transformation properties for the fields. In particular (with obvious notation):

$$
\left.\begin{array}{l}
\underset{\sim}{x}+\underset{\sim}{v} t=\underbrace{\prime}_{\sim}, \quad t=t^{\prime} \text { and } \\
\underset{\sim}{E^{\prime}}=\underset{\sim}{E}-\underset{\sim}{v} \times \underset{\sim}{H}-(\underset{\sim}{v} \cdot \underset{\sim}{x}) C U R L \underset{\sim}{H}+0\left(v^{2}\right), \\
\underset{\sim}{H^{\prime}}=\underset{\sim}{H}+\underset{\sim}{v} \times \underset{\sim}{E}+(\underset{\sim}{v} \cdot \underset{\sim}{x}) C U R L \underset{\sim}{E}+0\left(v^{2}\right) .
\end{array}\right\}
$$

This is in contradistinction to the Lorentz transformations, where:

$$
\begin{aligned}
& \underset{\sim}{x^{\prime}}=\underset{\sim}{x}+\underset{\sim}{v} t+0\left(v^{2}\right), \quad t^{\prime}=t+\underset{\sim}{v} \cdot \underset{\sim}{x}+0\left(v^{2}\right), \\
& \underset{\sim}{E^{\prime}}=\underset{\sim}{E}-\underset{\sim}{v} \times \underset{\sim}{H}+0\left(v^{2}\right), \\
& {\underset{\sim}{H}}^{\prime}=\underset{\sim}{H}+\underset{\sim}{v} \times \underset{\sim}{E}+0\left(v^{2}\right) .
\end{aligned}
$$


Since this result is so revolutionary and not well-known, we give a more precise formulation (however, we will find (A) and (B) useful later).

Let $\psi=\left[E_{1}, E_{2}, E_{3}, H_{1}, H_{2}, H_{3}\right]^{T}$ and $\mathbf{Q}=i\left[\begin{array}{cc}0_{3} & -I_{3} \\ I_{3}, & 0_{3}\end{array}\right]$, where $0_{3}$ and $I_{3}$ are $3 \times 3$ zero and unit matrices. Define $\underset{\sim}{S}$ by:

$$
S_{1}=\left[\begin{array}{ccc}
0 & 0 & 0 \\
0 & 0 & -i \\
0 & i & 0
\end{array}\right], S_{2}=\left[\begin{array}{ccc}
0 & 0 & i \\
0 & 0 & 0 \\
-i & 0 & 0
\end{array}\right] \text { and } S_{3}=\left[\begin{array}{ccc}
0 & -i & 0 \\
i & 0 & 0 \\
0 & 0 & 0
\end{array}\right]
$$

We now set $\mathbf{H}=i \mathbf{Q}(\underset{\sim}{S} \underset{\sim}{\nabla})$ so that Maxwell's equations can be written as:

$$
i \frac{\partial \psi}{\partial t}=\mathbf{H} \psi, \underset{\sim}{\nabla} \underset{\sim}{E}=0, \underset{\sim}{\nabla} \cdot \underset{\sim}{H}=0 .
$$

It is shown in [12] that the above system is invariant under the following two algebras (Poincare):

$$
\left.\begin{array}{l}
P_{\mu}=\frac{\partial}{\partial x_{\mu}}=P_{\mu}^{I}, \quad \dot{\mu}=0,1,2,3, \quad x_{0}=t \\
J_{a b}^{I}=x_{a} P_{b}-x_{b} P_{a}+i \epsilon_{a b c}\left(\begin{array}{ll}
S_{c} & 0 \\
0 & S_{c}
\end{array}\right), \quad 1 \leq a, b \leq 3, \\
J_{o a}^{I}=x_{o} P_{a}-x_{a} P_{0}+\mathbf{Q S}_{a} \\
J_{a b}^{I I}=J_{a, b}^{I}(1 \leq a, b \leq 3), \quad P_{0}^{I I}=-i \mathbf{H}, \quad P_{a}^{I I}=P_{a} \\
J_{o a}^{I I}=t P_{a}-\frac{i}{2}\left(\mathbf{H} x_{a}+x_{a} \mathbf{H}\right)+\frac{1}{2} \mathbf{Q} S_{a} .
\end{array}\right\}
$$

The operators $J_{o a}^{I}$ generate the Lorentz transformations associated with (B), while the operators $J_{o a}^{I I}$ generate the Galilean transformation associated with (A). The explicit Lie group generated by $J_{o a}^{I I}$ is:

$$
\begin{aligned}
& t^{\prime}=e^{t(\underset{\sim}{v} \underset{\sim}{\sim})} t e^{-t(v \underset{\sim}{v})}=t \\
& x_{\sim}^{\prime}=e^{t(\underset{\sim}{v \cdot \nabla})} \underset{\sim}{x} e^{-t(\underset{\sim}{v} \underset{\sim}{v})}=\underset{\sim}{x}+\underset{\sim}{v t}, \text { and } \\
& \psi^{\prime}\left(\underset{\sim}{x^{\prime}}, t\right)=\exp \{t(\underset{\sim}{v} \underset{\sim}{\nabla})\} \exp \{-t(\underset{\sim}{\nabla} \underset{\sim}{\nabla})+ \\
& \mathbf{Q}[\underset{\sim}{S} \cdot \underset{\sim}{v}+(\underset{\sim}{v} \underset{\sim}{x} \cdot)(\underset{\sim}{S} \cdot \underset{\sim}{\nabla})]\} \psi(\underset{\sim}{x}, t) .
\end{aligned}
$$


Returning to (A) and (B), we see that the complications in the transformation properties of the space-time variables in (B) provide for simple transformation properties for the field variables. On the other hand, the simple transformation properties of the space-time variables in (A) lead to more complex transformation properties for the field variables. The important physical issue is to determine which of the two is appropriate for a consistent and faithful representation of physical reality.

Although many of us believe we have a fairly comprehensive understanding of Lorentz transformations for space-time variables, the truth is that many of us were only familiarized with the transformations for $1+1$ or $1+3$ dimensions with collinear velocities. In fact, the Thomas rotation is an essential device for extending Lorentz transformations from $1+1$ to $1+3$ dimensions (see Ungar [13]). What is not so well-known (see [14]) is the fact that when the velocities are non-collinear, the relativistic (Lorentz) addition laws are not commutative nor associative and this in turn has been shown to lead to what is known as the Mocanu paradox after its discover [13]. In particular, $\underset{\sim}{v_{1}} \oplus \underset{\sim}{v_{2}} \neq v_{2} \oplus \underset{\sim}{v_{1}}$ for noncollinear $v_{1}$ and $v_{2}$, where $\oplus$ represents the Lorentz addition. It turns out that a continuous rotation (in time) is required to bring the two representations into equality. This of course implies the existence of a non-inertial aspect to this approach (see [13]). Of course, this problem and the Mocanu paradox dissappears when we consider the Fushchych-Shtelen transformation (A).

There are a host of other not so well-known problems and/or issues associated with the current implementation of special relativity. Santilli $([15],[16])$ has identified what he calls "real big paradoxes". These issues tend to focus directly on the speed of light postulate and, although very important, will not be discussed here (see also the list of problems identified by Sastri [17]).

\section{Proper-time formulation}

The dynamics of a classical observable can be conveniently studied by Hamiltonian mechanics using the Poisson bracket $\{A(p, q), B(p, q)\}=\frac{\partial A}{\partial p} \frac{\partial B}{\partial q}-\frac{\partial A}{\partial q} \frac{\partial B}{\partial p}$. The Hamilton equations ensure that the time development of an arbitrary classical function $W(q, p, t)$ is given by

$$
\frac{d W}{d t}(q, p, t)=\{H, W(q, p, t)\}+\frac{\partial W}{\partial t}(q, p, t) .
$$

Defining the proper time $\tau$ by $d t=\frac{H}{m c^{2}} d \tau$, the proper-time evolution of the function $W$ is given by the chain rule:

$$
\frac{d W}{d \tau}=\frac{d W}{d t} \frac{d t}{d \tau}=\frac{H}{m c^{2}}\{H, W\}+\frac{\partial W}{\partial \tau} .
$$

An energy functional $K$ which is conjugate to the proper-time $\tau$ will be defined by $\{K, W\}=\frac{H}{m c^{2}}\{H, W\}$ with $K=m c^{2}$ when $H=m c^{2}$. If the mass $m$ remains invariant during the evolution, this functional can be directly determined since

$$
\frac{H}{m c^{2}} \frac{\partial H}{\partial p} \cdot \frac{\partial w}{\partial q}-\frac{H}{m c^{2}} \frac{\partial H}{\partial q} \frac{\partial w}{\partial p}=\frac{\partial}{\partial p}\left[\frac{H^{2}}{2 m c^{2}}+\alpha\right] \frac{\partial w}{\partial q}-\frac{\partial}{\partial q}\left[\frac{H^{2}}{2 m c^{2}}+\alpha\right] \frac{\partial w}{\partial p},
$$


where $\alpha$ is a constant, leads to:

$$
K=\frac{H^{2}}{2 m c^{2}}+\frac{m c^{2}}{2}
$$

and the evolution of the function $\mathrm{W}$ in terms of $\tau$ can be expressed as

$$
\frac{d W}{d \tau}=\{K, W\}+\frac{\partial W}{\partial \tau} .
$$

The usual form of the Hamiltonian representing a single particle is $H=\sqrt{c^{2} P^{2}+\left(m c^{2}\right)^{2}}$. For this $\mathrm{H}$, the conjugate proper energy is given by $K=\frac{P^{2}}{2 m}+m c^{2}$. Thus, we see that the functional form of the energy $K$ is the same as that of the nonrelativistic energy of the system, even though the system is fully relativistic.

The above result shows that our approach preserves Hamilton's equations but does not necessarily represent a canonical transformation of variables. This means that we are not assured that a Lagrangian exists, etc.

Let $H=\sum_{i} H_{i}, M=\sum_{i} m_{i}$ be a closed interacting system with $H$ and $M$ constants with $M$ independent of particle variables. Let $\left\{p_{i}\right\},\left\{q_{i}\right\}$ and $t$ represent the momentum, position, and time variables of the system for some inertial observer.

DEFINITION 1.1. We say that the transformation on extended phase space which transforms $\left.\underset{\sim}{\left(p_{i}\right.}, q_{\sim}, t\right) \longrightarrow\left(\underset{\sim}{P_{i}}, \underset{\sim}{Q_{i}}, T\right)$ is a canonical (contact) transformation $(t \neq T)$ provided that there exist functions, $F=F\left(\underset{\sim}{P_{i}}, \underset{\sim}{Q_{i}}, T\right)$ and $K=K\left(\underset{\sim}{P_{i}}, Q_{\sim}, T\right)$ such that:

$$
\sum_{i} \underset{\sim}{p_{i}} \cdot d \underset{\sim}{q_{i}}-H d t=\sum_{i} \underset{\sim}{P_{i}} d Q_{\sim}-K d T+d F
$$

THEOREM 1.2. The transformation which maps $\left.\underset{\sim}{\left(p_{i}\right.}, \underset{\sim}{q_{i}}, t\right) \rightarrow\left(\underset{\sim}{p_{i}}, q_{i}, \tau\right)$ is a canonical contact transformation in the sense of Definition $1 . \tilde{1}$ (e.g., it preserves the PoincaréCartan invariant (1.3)).

PROOF. Since $d \tau=\frac{M c^{2}}{H} d t$, we can write:

$$
H d t=\frac{H^{2}}{M c^{2}} d \tau=\left(\frac{H^{2}}{2 M c^{2}}+\frac{M c^{2}}{2}\right) d \tau-\left(\frac{M c^{2}}{2}-\frac{H^{2}}{2 M c^{2}}\right) d \tau
$$

so that:

$$
H d t \equiv K d \tau-\left(\frac{M c^{2}}{2}-\frac{H^{2}}{2 M c^{2}}\right) d \tau
$$

It is clear from (1.3) that we are done if we can show that $\left(\frac{H^{2}}{2 M c^{2}}-\frac{M c^{2}}{2}\right) d \tau$ is a total differential of some function $F$. Since our system is closed by assumption, $H$ and $M$ are 
constants with $M$ independent of the particle variables. If we set $F=\left(\frac{H^{2}}{2 M c^{2}}-\frac{M c^{2}}{2}\right) \tau$, we have

$$
\begin{aligned}
\frac{d F}{d \tau} & =\sum_{i} \frac{\partial F}{\partial p_{i}} \underset{\sim}{d q_{i}} \frac{\partial F}{d \tau}+\underset{\sim}{\frac{d q_{i}}{\sim}}+\frac{\partial F}{\partial \tau} \\
& =\sum_{i}\left(\underset{\frac{d q_{i}}{\sim}}{\frac{d p_{i}}{d \tau}} \frac{d p_{i}}{d \tau}-\frac{d q_{i}}{d \tau} \frac{\sim}{d \tau}\right)+\left(\frac{H^{2}}{2 M c^{2}}-\frac{M c^{2}}{2}\right) \\
& =\left(\frac{H^{2}}{2 M c^{2}}-\frac{M c^{2}}{2}\right)
\end{aligned}
$$

Hence $d F=\left(\frac{H^{2}}{2 M c^{2}}-\frac{M c^{2}}{2}\right) d \tau$, so that $F$ is the generator of our transformation.

Let us now prove that our implementation satisfies the two postulates of special relativity. Assuming our system is in a vacumn, it follows from the definition of $\tau$ that "the speed of light is independent of the motion of the source".

First suppose we have a free particle and consider two inertial observers in frames $X, X^{\prime}$ with extended phase space coordinates $(\underset{\sim}{p}, \underset{\sim}{q}, t),\left(\underset{\sim}{p^{\prime}}, \underset{\sim}{q^{\prime}}, t^{\prime}\right)$ respectively. Let $P$ denote the set of Poincaré transformations on space-time reference frames, $P\left(X, X^{\prime}\right): X \rightarrow X^{\prime}$, and denote by $T$ the set of canonical proper-time transformations defined on extended phase space. We denote the map $(\underset{\sim}{p} \underset{\sim}{q}, t) \mapsto(\underset{\sim}{p} \underset{\sim}{q}, \tau)$ by $T(X, t, \tau)$. By Theorem $(1.2)$, we have that $T$ is a group.

THEOREM 1.3. The proper-time coordinates on $X$ are related to those on $X^{\prime}$ by the transformation:

$$
S_{m}\left(X, X^{\prime}, \tau\right)=T\left(X^{\prime}, t^{\prime}, \tau\right) P_{m}\left(X, X^{\prime}\right) T^{-1}(X, t, \tau) .
$$

PROOF. The proof follows from the commutativity of the following diagram.

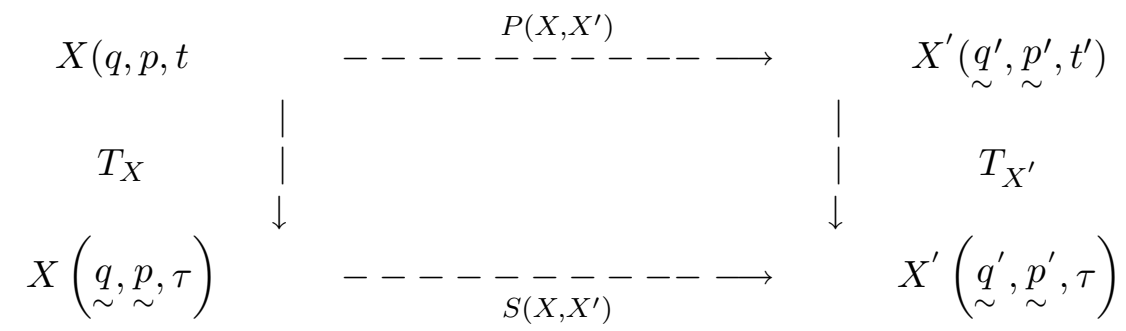

We have used the particle mass in the statement of the above theorem to fix the observed system. The group of proper-time transformations depends on 14 parameters $\left(m, \underset{\sim}{p} \underset{\sim}{q}, \underset{\sim}{p^{\prime}}, \underset{\sim}{q^{\prime}}, \tau\right)$. It follows that the free-particle laws will be the same for all inertial observers and will be form invariant under a similarity group action on the Poincaré group.

As will be discussed later, Theorem 1.3 is not true for interacting many-particle systems. However, if the particle is a photon and $t=t^{\prime}$ (Fuchchych-Shtelen), setting $m c^{2}=H$ 
and $H^{\prime}=m^{\prime} c^{2}$, where $m$ and $m^{\prime}$ represent the effective mass as seen in X and $\mathrm{X}$ ', shows that $T_{X}=T_{X^{\prime}}=I$ so that $S\left(X, X^{\prime}\right)=P\left(X, X^{\prime}\right)$.

COROLLARY 1.4. The Fushchych-Shtelen transformation is in $T$.

In the general case of Theorem 1.2, observers in $X$ and $X^{\prime}$ can still identify $\tau$ and hence can construct canonical variables

$$
\left.\left(K, \underset{\sim}{p_{i}}\right\},\{\underset{\sim}{q}\}, \tau\right) \text { and }\left(K^{\prime},\left\{\underset{\sim}{p_{i}^{\prime}}\right\},\left\{\underset{\sim}{q_{i}^{\prime}}\right\}, \tau\right) .
$$

It follows that if we represent $X$ and $X^{\prime}$ in configuration space and use the relationships (for suitably chosen origins of the spatial coordinate systems):

$\underset{\sim}{q_{i}^{\prime}}={\underset{\sim}{q}}_{\sim}^{\prime}-\underset{\sim}{u} \tau, \underset{d q_{i}^{\prime}}{\stackrel{\sim}{d \tau}}=\underset{\sim}{q_{i}}-\underset{\sim}{u}$, where $\underset{\sim}{u}$ is the relative velocity of the two frames; we see that these frames are basically connected by the Galilei group. To be precise, let $\hat{L}_{t}$ denote the Legendre transformation mapping phase space onto configuration space and let $\hat{X}_{\tau}$ denote the configuration space representation of $\widehat{X}_{\tau}$. If $G$ is the associated Galilean map, we have the following:

THEOREM 1.5. The proper-time coordinates on $X$ are related to those on $X^{\prime}$ by the transformation

$$
M\left(X, X^{\prime}, \tau\right)=T\left(X^{\prime}, \tau\right)^{-1}\left(\widehat{L}_{\tau}^{\prime}\right)^{-1} G\left(\widehat{L}_{\tau}\right) T(X, \tau) .
$$

PROOF. The proof follows from the commutativity of the following diagram:

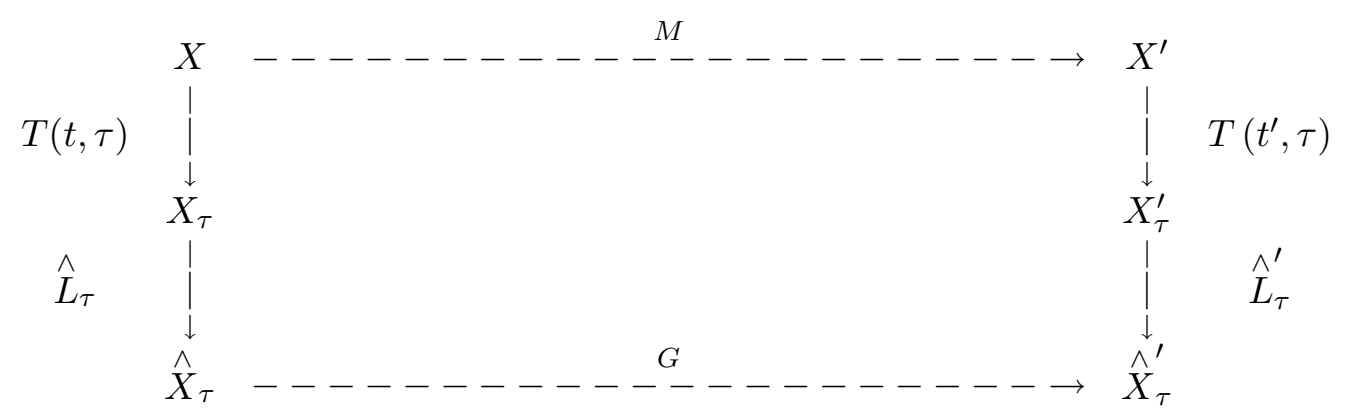

We can now state the following theorems:

THEOREM 1.6. There exists a unique clock for the time-observation of any closed interacting relativistic system.

THEOREM 1.7. There is a many-particle direct-interaction theory with the following properties:

1. the theory satisfies the first two postulates of special relativity;

2. the theory is based on Hamiltonian dynamics;

3. the theory is based on independent (canonical) particle variables.

Theorem 1.6 is implicit in our construction of $K, K^{\prime}$ and $\tau$ for the many-particle system. Theorem 1.7 follows from Theorem 1.5, but we made it explicit since the following theorem is known when one replaces our first condition by the requirement of Lorentz invariance. In particular, we have: 
THEOREM 1.8. Suppose we seek a many-particle direct-interaction theory with the following properties:

1. the theory is Lorentz invariant,

2. the theory is based on a Hamiltonian dynamics, and

3. the theory is based on independent (canonical) particle variables.

Then such a theory is only compatible with noninteracting particles.

PROOF. The proof of this result is well-known and can be found in many places (see Mann [18]). It was first proved by Currie [7].

Theorem 1.8 is known as the "no-interaction" theorem. It has been a major (if not the major) impediment to the successful merging of special relativity with quantum mechanics (cf. Rohrlich [3]). Theorem 1.7 shows that if we abandon the third postulate of Minkowski and use the clock determined by the proper-time for the system dynamics along with its canonical Hamiltonian, we obtain a theory which completely avoids the no-interaction difficulties.

It follows from Theorem 1.8 that the map $M$ in Theorem 1.5, is not a Poincaré map nor is it necessarily a Galilean map. The set of such maps form a group which clearly deserves independent study.

\section{Interaction}

Prior to studying more closely the case of interactions, let us review the essential ideas concerning Santilli's Lie-isotopes [15] and their properties. For a complete review see Kadeisvilli [19]. Let $G$ denote a given Lie algebra with bracket $[A, B]=A B-B A$ and let $T$ be an invertible element in $G$. A Lie-isotope of $G$ is then defined as $G$ with the bracket $[A, B]^{*}=A * B-B * A \equiv A T B-B T A$. It is easy to show that $[,]^{*}$ is a Lie bracket and that $\left(G,[,]^{*}\right)$ is a Lie algebra. It turns out that two nonisomorphic groups may have isotopic Lie algebras. The standard example concerns the groups $\mathrm{SO}(3), \mathrm{SO}(2,1)$. These are symmetry groups for the following respective Hamiltonians:

$$
\begin{aligned}
& H_{1}(\underset{\sim}{q} \underset{\sim}{p})=\frac{1}{2}\left(p_{1}^{2}+p_{2}^{2}+p_{3}^{2}\right)+\frac{1}{2}\left(q_{1}^{2}+q_{2}^{2}+q_{3}^{2}\right), \\
& H_{2}(\underset{\sim}{q} \underset{\sim}{p})=\frac{1}{2}\left(p_{1}^{2}-p_{2}^{2}+p_{3}^{2}\right)+\frac{1}{2}\left(q_{1}^{2}-q_{2}^{2}+q_{3}^{2}\right) .
\end{aligned}
$$

These Hamiltonians lead to the same equations of motion and to the same conservation laws (via Noether's theorem) for the components of angular momentum $L_{b}(b=1,2,3)$.

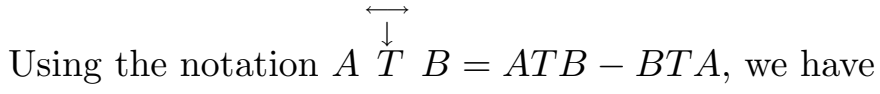

$$
\left[L_{b}, L_{c}\right]=\frac{\partial L_{b}}{\partial q_{i}} \stackrel{\stackrel{\downarrow}{\delta_{j}^{i}}}{{ }_{j}} \frac{\partial L_{c}}{\partial p_{j}}, \quad\left[\delta_{j}^{i}\right]=\left[\begin{array}{ccc}
1 & 0 & 0 \\
0 & 1 & 0 \\
0 & 0 & 1
\end{array}\right],
$$

and

$$
\left[L_{b}, L_{c}\right]^{*}=\frac{\partial L_{b}}{\partial q_{i}} \stackrel{\overleftrightarrow{\downarrow}}{\alpha_{j}^{i}} \frac{\partial L_{c}}{\partial p_{j}}, \quad\left[\alpha_{j}^{i}\right]=\left[\begin{array}{ccc}
1 & 0 & 0 \\
0 & -1 & 0 \\
0 & 0 & 1
\end{array}\right]
$$


for the respective Lie algebra of the groups $\mathrm{SO}(3)$ and $\mathrm{SO}(2,1)$. In the latter case we have $T=\left[\alpha_{j}^{i}\right]=T^{-1}$.

In order to understand the requirement that $T$ be invertible, recall that the group $\mathrm{SO}(3)$ leaves the standard inner product $<a, b>_{3}=a_{1} b_{1}+a_{2} b_{2}+a_{3} b_{3}$ invariant while the group $\mathrm{SO}(2,1)$ leaves $<\underset{\sim}{a}, \underset{\sim}{b}>_{2,1}=a_{1} b_{1}-a_{2} b_{2}+a_{3} b_{3}$ invariant. We can write $<\underset{\sim}{a}, \underset{\sim}{b}>_{3}=$ $(u \underset{\sim}{u})^{t} I(\underset{\sim}{u b})=(\underset{\sim}{a})^{t} I(\underset{\sim}{\underset{\sim}{\sim}})=(\underset{\sim}{a})^{t}(\underset{\sim}{b})$ so that $u^{t} I u=I$ if $u^{t}=u^{-1}, u \epsilon S O(3) ;$ while $<\underset{\sim}{a}, \underset{\sim}{b}>_{2,1}=(\underset{\sim}{u})^{t} \tilde{I}(u \underset{\sim}{b})=(\underset{\sim}{a})^{t} \tilde{I}(\underset{\sim}{\sim})$ if $u^{t} \tilde{I} u=\tilde{I}$ for $u \epsilon S O(2,1)$ with $\widetilde{I}=T^{-1}$.

It can be shown that by the above process of changing the binary operation of multiplication along with a redefinition of the unit, all of algebra, geometry and analysis can be generalized in a consistent manner. Santilli [19] conceived of isotopes as a means of providing the mathematical structure to study physics in a non-vacuum-like medium, where, in general, the special theory need not hold. For example, inside a proton or star. It should be noted that even in water, one implication of special relativity fails; namely, that the maximal velocity is the speed of light (e.g. Cherenkov radiation). More unnerving is the fact that the velocity sum rule does not apply (see [16]).

It is generally believed that the problem of interaction was resolved when the potential energy was found to fit perfectly as the scalar component of a four-vector and led to the "Principle of Minimal Interaction". A detailed study and classification of the problems with this approach can be found in the recent book by Fushchych and Niktin [21]. It turns out that only for relativistic wave equations with spin $s \leq \frac{1}{2}$ can one be assured that no inconsistencies will occur. For $s \geq 1$, we have the following types of problems (see [20] pg. 117 for details):

1. The equation becomes inconsistent.

2. The equation acquires redundant components making it impossible to interpret as an equation for a spin s particle.

3. The equations describe faster-then-light propagation $(s>1$.)

4. The equations become inconsistent when applied to concrete problems.

The principle has its roots in the observation that the potential energy fits perfectly as the fourth component of a four-vector. The four-vector is not required for the implementation of our approach. In fact, a detailed analysis of the symmetry of the Dirac equation with minimal interaction has led us to treat the potential energy as a Lorentz scalar (e.g., a part of the mass). This work is in preparation and will be reported elsewhere.

In light of the above, we consider the following two Hamiltonians:

Case 1. $\quad H=\left[c^{2} p^{2}+\left(m c^{2}+V\right)^{2}\right]^{1 / 2}$,

Case 2. $\quad H=\left[c^{2} p^{2}+m^{2} c^{4}\right]^{1 / 2}+V$,

corresponding to two different ways of describing particle interactions. Here, $V$ is assumed to be independent of the momenta.

In case 1 we obtain

$$
\frac{d q}{d t}=\frac{c^{2}}{H} p, \quad \frac{d t}{d \tau}=\frac{H}{m c^{2}+V},
$$




$$
\frac{d p}{d t}=\frac{\left(m c^{2}+H\right)}{H}(-\underset{\sim}{\nabla} V)
$$

so that

$$
\underset{\sim}{\stackrel{d q}{d \tau}}=\left(m+\frac{V}{c^{2}}\right)^{-1} \underset{\sim}{p}
$$

and

$$
\frac{d p}{d \tau}=-\underset{\sim}{\nabla} V
$$

We impose the condition that $K$ be of the same form when interaction is introduced. This implies that (as in 1.1), we have: $\frac{d W}{d \tau}=\frac{H}{m c^{2}+V}\{H, W\}$ or

$$
\frac{d W}{d \tau}=\frac{\partial K}{\partial p} T \underset{\sim}{\frac{\partial W}{\partial q}}-\underset{\sim}{\frac{\partial K}{\partial q}} T \frac{\partial W}{\partial \underset{\sim}{p}}=\{K, W\}^{*}
$$

Thus, our requirement shows that interaction induces a change in the internal symmetry structure of the system described by an isotope of the free particle algebra. Note from (2.1) that for $\frac{V}{c^{2}} \ll 1$, we get $T \sim I$ (e.g., the non-relativistic approximation).

For case 2 we obtain

$$
\begin{aligned}
& \frac{d \underset{\sim}{q}}{d t}=\frac{c^{2} \underset{\sim}{\sim}}{H-V}, \quad \quad \stackrel{d \underset{\sim}{p}}{d t}=-\underset{\sim}{\nabla} V, \\
& \frac{d t}{d \tau}=\frac{H-V}{m c^{2}}=\frac{H}{m c^{2}}\left(1-\frac{V}{H}\right), \\
& \frac{d q}{d \tau}=\frac{\underset{\sim}{p}}{m}, \quad \frac{d p}{d \tau}=\left(\frac{H-V}{m c^{2}}\right)(-\underset{\sim}{\nabla} V),
\end{aligned}
$$

and the analogue of (2.3):

$$
\frac{d W}{d \tau}=\frac{H}{m c^{2}}\left[\frac{\partial H}{\partial \underset{\sim}{p}}\left(1-\frac{V}{H}\right) \frac{\partial W}{\partial \underset{\sim}{q}}-\frac{\partial H}{\partial \underset{\sim}{q}}\left(1-\frac{V}{H}\right) \frac{\partial W}{\partial \underset{\sim}{p}}\right] .
$$

In the present case we set $T=1-\frac{V}{H}$ and $\tilde{I}=T^{-1}$ so that $T=T^{-1} \cong I$ in the region $V \ll H$. $K$ is given by (1.1), and we find

$$
K=\frac{p^{2}}{2 m}+m c^{2}+V \sqrt{1+\left(\frac{p}{m}\right)^{2}}+\frac{V^{2}}{2 m c^{2}} .
$$

For purposes of comparison, we note that for case 1,

$$
K=\frac{p^{2}}{2 m}+m c^{2}+V+\frac{V^{2}}{2 m c^{2}} .
$$


We note that the two Hamiltonians (2.5), (2.6) agree in the nonrelativistic limit but differ from each other in the ultrarelativistic regime.

\section{Many-particle theory}

We now suppose that we have a system of $N$ particles. We assume that the Hamiltonian for each particle may be written as:

$$
\begin{aligned}
H_{j} & =\sqrt{c^{2} \pi_{j}^{2}+\left(m_{j} c^{2}+V_{j}\right)^{2}} \\
H & =\sum_{j} H_{j} \\
M & =\sum_{j} m_{j} \\
\pi_{j} & =\left(\underset{\sim}{p_{j}}-\frac{e}{c} A_{j}\right) .
\end{aligned}
$$

Set $\bar{m}_{j}=m_{j}+V_{j} / c^{2}$, so that:

$$
\left.\begin{array}{rl}
d \tau_{j} & =\frac{\bar{m}_{j} c^{2}}{H_{j}} d t \\
d \tau & =\frac{M c^{2}}{H} d t .
\end{array}\right\}
$$

We are assuming that we have a closed system so that $H$ is conserved. Furthermore, we assume that:

$$
\left.\begin{array}{l}
\underset{\sim j}{A}=\frac{1}{2} \sum_{i \neq j} A_{i j}\left(\left|\underset{\sim}{q_{j}}-\underset{\sim}{q_{i}}\right|, \tau\right) \\
V_{j}=\frac{1}{2} \sum_{i \neq j} V_{i j}\left(\left|\underset{\sim}{q_{j}}-\underset{\sim}{q_{i}}\right|, \tau\right) .
\end{array}\right\}
$$

This means that we restrict ourselves to directly interacting particles, although the addition of fields causes no problems.

If $W$ is any function of the $\left\{\underset{\sim}{p_{j}}, q_{j}\right\}$ then:

$$
\frac{d W}{d t}=\sum_{j}\left(\underset{\sim}{\frac{\partial H}{\partial p_{j}}} \underset{\sim}{\frac{\partial W}{\partial q_{j}}}-\frac{\partial H}{\partial p_{j}} \underset{\sim}{\frac{\partial W}{\partial p_{j}}}\right)=\{H, W\} .
$$

Using (3.1) we obtain:

$$
\frac{d W}{d \tau}=\frac{H}{M c^{2}}\{H, W\}=\{K, W\},
$$


where $K=\frac{H^{2}}{2 M c^{2}}+\frac{M c^{2}}{2}$. We can also write (3.2) as:

$$
\begin{aligned}
\frac{d W}{d \tau}=\frac{H}{M c^{2}} \sum_{i}\left\{H_{i}, W\right\} & =\sum_{i} \frac{H}{M c^{2}} \frac{\bar{m}_{i}}{H_{i}}\left(\frac{H}{\bar{m}_{i}}\left\{H_{i}, W\right\}\right) \\
& =\sum_{i} \frac{d \tau_{j}}{d \tau} \frac{H_{i}}{\bar{m}_{i} c^{2}}\left\{H_{i}, W\right\},
\end{aligned}
$$

where we have used $\frac{d \tau_{i}}{d \tau}=\frac{H}{M} \frac{\bar{m}_{i}}{H_{i}}$, which follows from (3.1). We now use the results of Section 2 to obtain for each i:

$$
\begin{aligned}
\frac{H_{i}}{\bar{m}_{i} c^{2}}\left\{H_{i}, W\right\} & =\frac{m_{i}}{\bar{m}_{i}}\left\{K_{i} W\right\} \\
& =\sum_{j} \frac{\partial K_{i}}{\partial p_{j}}\left(\frac{m_{i}}{\bar{m}_{i}}\right) \frac{\partial W}{\partial q_{j}}-\frac{\partial K_{i}}{\partial q_{j}}\left(\frac{m_{i}}{\bar{m}_{i}}\right) \frac{\partial W}{\partial p_{j}}=\left\{K_{i}, W\right\}_{i}^{*} .
\end{aligned}
$$

Combining (3.4) with (3.3), we have:

$$
\frac{d W}{d \tau}=\sum_{i} \frac{d \tau_{i}}{d \tau}\left\{K_{i}, W\right\}_{i}^{*}
$$

where $K_{i}=\frac{H_{i}^{2}}{2 m_{i} c^{2}}+\frac{m_{i} c^{2}}{2}$, and $\left\{K_{i}, \cdot\right\}_{i}^{*}=\sum_{j} \frac{\partial K_{i}}{\partial p_{j}}\left(\frac{m_{i}}{\bar{m}_{i}}\right) \frac{\partial}{\partial q_{j}}-\frac{\partial K_{i}}{\partial q_{j}}\left(\frac{m_{i}}{\bar{m}_{i}}\right) \frac{\partial}{\partial p_{j}}$.

Suppose our system of $N$ particles separates into $N_{1}+\tilde{N}_{2}$ particles. We assume that the $N_{1}$ particles are sufficiently distant from the $N_{2}$ particles that all inertial observers can identify the two clusters. We associate with each cluster total Hamiltonians $H^{1}, H^{2}$ and total masses $M_{1}, M_{2}$. Although it is not necessary, we assume for simplicity that $M_{1}, M_{1}$ are constants independent of the particle variables. If this is not the case, we simply use the Lie-isotopic methods of Section 2. We can now write

$$
d \tau_{1}=\frac{M_{1} c^{2}}{H_{1}} d t, d \tau_{2}=\frac{M_{2} c^{2}}{H^{2}} d t
$$

and derive the following dynamical equations for the individual systems:

$$
\frac{d W_{1}}{d \tau_{1}}=\left\{K_{1}, W_{1}\right\}^{1}, \frac{d W_{2}}{d \tau_{2}}=\left\{K_{2}, W_{2}\right\}^{2}
$$

where $\left\{K_{i},\right\}^{i}=\sum_{j}^{N_{i}}\left(\underset{\sim}{\frac{\partial K_{i}}{\partial p_{i j}}} \cdot \frac{\partial}{\partial q_{i j}}-\frac{\partial K_{i}}{\partial q_{i j}} \frac{\partial}{\partial p_{i j}}\right)$. This gives us a natural description of the cluster decomposition. It should be noted that, even if each of the above clusters is closed and noninteracting, there still exists a global system which connects them with a Hamiltonian given by $H=H^{1}+H^{2}$ and a mass given by $M=M_{1}+M_{2}$. This observation leads to the following:

THEOREM 3.1. Suppose we assume that inertial observers always choose frames of reference in which they are at rest relative to the observed system. Then there exists a unique (up to position of origin) rest-frame for the universe. 
PROOF. Let $U_{1}$ denote an arbitrary subset of the universe, let observer $0_{1}$ be at rest relative to $U_{1}$, and let $U_{2}$ denote the complement of $U_{1}$. $U_{1}$ and $U_{2}$ each have proper times $\tau_{1}, \tau_{2}$ along with masses $M_{1}, M_{2}$ and Hamiltonians $H_{1}, H^{2}$. It follows that our observer can construct $M=M_{1}+M_{2}, H=H^{1}+H^{2}$ and $d \tau=\frac{M c^{2}}{H} d t$ along with $K=\frac{H^{2}}{2 M c^{2}}+\frac{M c^{2}}{2}$. It is clear that both $\tau$ and $K$ are unique and, since any observer can choose a frame in which he is at rest relative to the universe, all frames similarly chosen by other observers can only differ by the choice of the origins of the corresponding coordinate systems (which cannot affect the dynamics).

It is clear that no preferred reference is implied by the above result. On the other hand, we routinely use the distant stars as fixed for purposes of measurement.

\section{Conclusion}

We have constructed an alternate approach to the implementation of special relativity. There are a number of implications and/or interesting issues to be explored in addition to conducting a comparative analysis with the Minkowski implementation. For example, if we replace $H \rightarrow-H, K$ remains the same while $\tau \rightarrow-\tau$. This means that there is a natural arrow of time associated with this approach and suggests an alternate explanation as opposed to the thermodynamic explanation.

At the basic level, note that the term $\frac{V^{2}}{2 m c^{2}}$ in $K$ is always repulsive. This suggests that a gravitational attraction can never lead to singularities. It also suggests (at a quantum level) that the discrete eigenvalues may be no more than averages caused by electron bound states in the potential well created by $V$ and $\frac{V^{2}}{2 m c^{2}}$. In the case of hydrogen, we can write $\frac{V^{2}}{2 m c^{2}}=\frac{1}{2}\left(\frac{r_{e}}{r}\right) \frac{e^{2}}{r}$, where $r_{e}=\frac{e^{2}}{m c^{2}}$ is the classical electron radius. Of course, such conjectures require a detailed study and analysis before anything definite can be said.

\section{Acknowledgment}

We sincerely thank W. Fushchych for his continued interest and support. This work is a result of many years of interaction with and support from R.M. Santilli. He has provided references, advanced copies of papers and books, in addition to providing continuous encouragement.

\section{References}

[1] Gill T.L. and Lindesay J., Int. J. Theo. Phys., 1993, V.32, 2087.

[2] Pryce M.H.L., Proc. Roy. Soc. London Ser. A, 1948, V.195, 400.

[3] Rohrlich F., Ann. Phys. (N.Y.), 1979, V.117, 292.

[4] Dirac P.A.M., Mathematical Foundations of Quantum Theory, Academic Press, New York, 1977.

[5] Rosen G., Foundations of Classical and Quantum Dynamical Systems, Academic Press, New York, 1969.

[6] Goldstein H., Classical Mechanics, Addison-Wesley, Reading, MA, 1950. 
[7] Currie D.G., J. Math. Phys., 1963, V.4, 1470;

Currie D.G., Jordan T.F. and Sudarshan E.C.G., Rev. Mod. Phys., V.35, 350.

[8] Gill T.L., Lindesay J., Zachary W.W. and Mahmood M.F., Hadronic J., V.17, 449.

[9] Whitney C.K., Physics Essays, 1989, V.2, 47.

[10] Whitney C.K., Hadronic Journal, 1988, V.11, 257.

[11] Whitney C.K., Galilean Electrodynamics, 1991, V.2, 28.

[12] Fushchych W.I. and Shtelen W.M., Dokl. AN Ukrain. SSR, 1991, N 3, 23-27.

[13] Ungar A.A., Found. Phys., 1989, V.19, N 11, 1385-1396.

[14] Landau L. and Lifshitz E., Mechanics, Pergamon Press, Oxford, 1968.

[15] Santilli R.M., Hadronic J., 1978, V.1, 223 and 1279.

[16] Santilli R.M., Isotopic Generalizations of Galilis and Einstein's Relativities, V.II: Classical Isotopes, Hadronic Press, Palm Harbor, Fl, 1991.

[17] Sastri G.P., Am. J. Phys., 1987, V.55, 943-945.

[18] Mann R.A., The Classical Dynamics of Particles, Academic Press, New York, 1974.

[19] Kadeisvili J.D., Santilli's Isotopes of Contemporary Algebras, Geometries and Relativities, Hadronic Press, Palm Harbor, Fl, 1992.

[20] Fushchych W.I., Shtelen W.M. and Serov N.I., Symmetry Analysis and Exact Solutions of Equations of Nonlinear Mathematical Physics, Kluwer Academic Publishers, Boston, 1993.

[21] Fushchych W.I. and Nikitin A.G., Symmetry of the Equations of Quantum Mechanics, Allenton Press, 1994.

[22] Fushchych W.I. and Nikitin A.G., Symmetries of Maxwell's Equations, D. Reidel, Dordrechet, 1987.

[23] Lorentz H.A., Collected Papers, The Hague Nijhoff, 1939, V.5, 330.

[24] Ritz W., Physikalische Zeitschrift, 1912, V.13, 317.

[25] Tolman R.C., Phys. Rev., 1910, V.31, 26.

[26] Poincare H., C.R. Acad. Sci. Paris, 1905, V.140, 1504.

[27] Gill T.L., Lindsay J., Zachary W.W., Mahmood M.F. and Chachere G., Proceedings of the International Workshop on New Frontiers in Relativity, Ed. by T.L. Gill, Hadronic Press, Palm Harbor, FL, 1996. 